\title{
The complex hybrid origins of the root knot nematodes revealed through comparative genomics
}

Root knot nematodes (RKN) can infect most of the world's agricultural crop species and are among the most important of all plant pathogens. As yet however we have little understanding of their origins or the genomic basis of their extreme polyphagy. The most damaging pathogens reproduce by obligatory mitotic parthenogenesis and it has been suggested that these species originated from interspecific hybridizations between unknown parental taxa. We have sequenced the genome of the diploid meiotic parthenogen Meloidogyne floridensis, and use a comparative genomic approach to test the hypothesis that this species was involved in the hybrid origin of the tropical mitotic parthenogen Meloidogyne incognita. Phylogenomic analysis of gene families from $M$. floridensis, $M$. incognita and an outgroup species Meloidogyne hapla was carried out to trace the evolutionary history of these species' genomes, and we demonstrate that $M$. floridensis was one of the parental species in the hybrid origins of $M$. incognita. Analysis of the $M$. floridensis genome itself revealed many gene loci present in divergent copies, as they are in $M$. incognita, indicating that it too had a hybrid origin. The triploid $M$. incognita is shown to be a complex doublehybrid between $M$. floridensis and a third, unidentified, parent. The agriculturally important RKN have very complex origins involving the mixing of several parental genomes by hybridization and their extreme polyphagy and success in agricultural environments may be related to this hybridization, producing transgressive variation on which natural selection can act. It is now clear that studying RKN variation via individual marker loci may fail due to the species' convoluted origins, and multi-species population genomics is essential to understand the hybrid diversity and adaptive variation of this important species complex. This comparative genomic analysis provides a compelling example of the importance and complexity of hybridization in generating animal species diversity more generally. 


\section{Authors}

2 David H Lunt ${ }^{1 *}$, Sujai Kumar², Georgios Koutsovoulos², Mark L Blaxter²,3

3 'School of Biological, Biomedical and Environmental Sciences, University of Hull, Hull, HU6 $4 \quad 7 R X$, UK

$5 \quad{ }^{2}$ Institute of Evolutionary Biology, University of Edinburgh, Edinburgh, UK

$6{ }^{3}$ The GenePool Genomics Facility, School of Biological Sciences, University of Edinburgh, 7 Edinburgh, UK

8 *Corresponding author: $\mathrm{Dr} \mathrm{DH}$ Lunt, School of Biological, Biomedical and Environmental 9 Sciences, University of Hull, Hull, HU6 7RX, UK +44 (0)1482 465514 dave.lunt@gmail.com 


\section{Introduction}

11 Root-knot nematodes (RKN) belong to the genus Meloidogyne, contain approximately 100

12 described species, and are globally important crop pathogens (Moens et al. 2009). The most

13 frequent, widespread, and damaging species ( $M$. incognita, $M$. arenaria, and $M$. javanica) are

14 tropical RKN that are highly polyphagous, infecting crop species producing the majority of the 15 world's food supply, with the damage attributable to RKN $\sim 5 \%$ of world agriculture (Taylor \& 16 Sasser 1978; Trudgill \& Blok 2001; Sasser \& Carter 1985). The adaptive phenotypic diversity of 17 these pathogens is also remarkable, with great variability observed both within and between 18 species with respect to host range and isolate-specific vulnerability to control measures (Trudgill

19 \& Blok 2001; Castagnone-Sereno 2006). The tropical RKN typically reproduce by obligatory 20 mitotic parthenogenesis and possess aneuploid genomes (Triantaphyllou 1982; Triantaphyllou 21 1985). These species have previously been suggested to be hybrid taxa, and phylogenetic 22 analysis of nuclear loci supports this conclusion (Dalmasso \& Berge 1983; Triantaphyllou 1985; 23 Hugall et al. 1999; Castagnone-Sereno 2006; Lunt 2008).

24 Hybrid speciation has a long history of study in plants, with hybrid species formation having had 25 a very significant influence on our understanding of species formation, diversity, and adaptation 26 (Arnold 1997; P. S. Soltis \& D. E. Soltis 2009). By contrast hybridization has been thought to be 27 much less common in animals, though the utilization of multilocus genetics, and more recently 28 genomics, has increased interest in the consequences of animal hybridization and several 29 reviews suggest that it is much more common and important than previously thought (Mallet 30 2007; Mallet 2005; Bullini 1994; Nolte \& Tautz 2010; Schwenk et al. 2008; Seehausen 2006).

31 Although there have been repeated suggestions that the tropical ("Group 1") RKN might have 32 hybrid origins, the parental species involved have never been identified. The phylogenies in 33 Hugall et al. (1999) and Lunt (2008) indicate that these parents (as represented by divergent 34 sequence clusters within the apomictic RKN) are more closely related to each other than either 
35 is to M. hapla, though neither had a parental species within their sampling schemes.

36 Meloidogyne floridensis is a plant pathogenic root knot nematode that was originally

37 characterized as $M$. incognita, but has since been described as a separate species on the basis

38 of its morphology and a unique esterase isozyme pattern (Jeyaprakash et al. 2006; Handoo et

39 al. 2004). Despite both nuclear rRNA and mtDNA sequences placing it within the phylogenetic

40 diversity of the tropical mitotic parthenogen (apomict) species (Tigano et al. 2005; Holterman et

41 al. 2009) (Figure 1), M. floridensis is a meiotic parthenogen (automict) with the standard 42 chromosome count of the meiotically reproducing RKN species $(n=18)$, has bivalent 43 chromosomes, and an observable meiotic division (Handoo et al. 2004). M. floridensis appears 44 to suppress the second meiotic division which is a known form of automictic reproduction called 45 first-division restitution and a pathway by which parental heterozygosity can be maintained (Bell 461982 p40). With the exception of M. floridensis, all of the Group 1 RKN (De Ley et al. 2002; 47 Holterman et al. 2009) are apomicts, unable to reproduce by meiosis, lacking bivalents, and 48 exhibiting extensive aneuploidy. This phylogenetic distribution of reproductive modes, with $M$. 49 floridensis phylogenetically nested within the diversity of the apomict RKN (Figure 1), is 50 unanticipated as it implies the physiologically unlikely route of re-emergence of meiosis from 51 within the obligate mitotic parthenogens. An alternative explanation for these observations is that 52 the observed phylogenetic relationships have not arisen from a typical ancestor-descendent 53 bifurcating process, but instead have been shaped by reticulate evolution and transfer of genes 54 by interspecific hybridization with $M$. floridensis a parent of the tropical apomict species.

\section{The origins of Meloidogyne incognita genomic duplicates}

56 The $M$. incognita genome revealed that many of the genes of this species are present as highly 57 divergent copies (Abad et al. 2008), a situation that seems to apply to the other tropical apomicts 58 too (Lunt 2008), though the origin of these divergent copies is controversial. One possible way to 59 account for the high divergence between alleles is that they have originated by a process of 60 'endoduplication' (Figure 2A). Here we use endoduplication to refer to two distinct processes, 
61 although their genomic outcomes are similar. Firstly, the entire $M$. incognita genome might have

62 doubled to become tetraploid. The homologous chromosomes may have then diverged, and the

63 extant pattern of partial retention of duplicated loci could be the result of gene loss. This process

64 would leave many areas of the genome possessing divergent copies. Second, an alternative mechanism possible in apomictic species such as $M$. incognita, is that former alleles that are released from the homogenizing effects of recombination, can independently accumulate mutations over long periods of time resulting in highly divergent homologous loci ('alleles') within a diploid genome (White 1945 pg 283; Judson \& Normark 1996).

Another possible explanation for a genome containing divergent homologous copies of many genes is interspecific hybridization. One (homeologous) copy is inherited from each parental species and the divergence between them derives from the divergence between the hybridizing taxa. It is likely here that all genes would be present as divergent copies, although gene conversion and related processes could homogenize some copies. If it originated by this second mechanism the resulting $M$. incognita genome would be a mosaic with genomic regions derived 75 from both its parents.

76 There are several ways in which $M$. incognita and $M$. floridensis might be related through hybridization. M. floridensis might be one of the two parental species which hybridized to form the tropical apomicts, including $M$. incognita (Figure 2B). Alternatively, M. floridensis might be an independent hybrid that shares one parental taxon with $M$. incognita, and thus represents a 'sibling' hybrid taxon (Figure 2C). Finally, M. floridensis may itself be a hybrid, but still have played a role as a parent of $M$. incognita by a subsequent hybridization event (Figure 2D). This last option predicts three gene copies in M. incognita and two in M. floridensis.

83 The nuclear gene phylogenies of Lunt (2008) indicate that the parental taxa of the apomict RKN

84 were closely related and derived from within the cluster of Group 1 Meloidogyne species after 85 the divergence of $M$. enterolobii (=M. mayaguensis). Since this closely matches the phylogenetic position of $M$. floridensis, which is known to reproduce via sexual recombination as the parental 
species also must have done, we set out to test by comparative genome sequencing and analysis if $M$. floridensis was one of the progenitors of the tropical apomicts.

89

\section{Reproductive mode and Meloidogyne evolutionary history}

Given the unexpected distribution of meiosis across Group 1 Meloidogyne species described above (Figure 1), there are several possible evolutionary pathways for the evolution of reproductive modes (Figure 2): In scenario 1, M. floridensis has regained meiosis from an apomict state. Alternatively (scenario 2), the numerous apomict species could have lost meiosis many times independently. There are several additional scenarios involving hybrid origins. In scenario 3 , the apomicts have hybrid origins with the automict $M$. floridensis as a putative parent, while in scenario 4 both $M$. floridensis and the apomicts have independent hybrid origins. In scenario 5, a hybrid $M$. floridensis is in turn parental to a complex hybrid apomict.

Scenario 1 is very unlikely. Meiosis is an exceptionally complex system to re-evolve once it has been lost (Dollo's law), and the only suggested example we are aware of in the literature is not supported by robust reanalysis (see (Goldberg \& Igić 2008)). In addition, the extant apomicts are highly aneuploid, making it necessary for $M$. floridensis to have re-evolved 18 homologous chromosome pairs, which again suggests that cytologically this route is highly unlikely. Scenario 2 is also not parsimonious, potentially implying very many independent major reproductive transitions. Since there are already genetic data indicating that the apomicts may have hybrid genomes (Lunt 2008), we focused our analyses on the much more biologically plausible scenarios 3, 4 and 5 that propose hybridization drove the evolution of the apomictic RKN.

Scenario 3 restricts the hybrid taxa to the apomict Group 1 species, and places $M$. floridensis as one of the hybridizing parental species (Figure $2 \mathrm{~B}$ ). This model makes predictions that, where divergent homeologous sequences are detected in the $M$. incognita genome, $M$. floridensis would possess two alleles closely related to one of these homeologues. The M. floridensis genome itself would also be substantially different from that of $M$. incognita, not possessing 
112 divergent homeologous blocks but rather displaying normal allelic variation, perhaps more

113 similar to that observed in the M. hapla genome.

114 In scenarios 4 (Figure 2C) and 5 (Figure 2D) M. floridensis would also be a product of an 115 interspecific hybridization, as are the apomicts. Both these scenarios predict that the $M$. 116 floridensis genome will, like $M$. incognita, show substantial sequence divergence between 117 homeologues throughout its genome, although it may also possess some regions where one 118 parental copy has been eliminated, and remaining diversity is simple allelism. In scenario 4, the 119 parents of $M$. incognita need not be the same as those of the apomicts, although the 120 phylogenetic position of $M$. floridensis implies that at least one of them may have been identical 121 or very closely related. The different putative hybrid origins of $M$. incognita predict two (scenario 122 4, Figure 2C) or three (scenario 5, Figure 2D) homeologous copies, potentially modified by 123 subsequent loss events.

124 Here, we generate a de novo assembled genome for $M$. floridensis, identify and analyse a large 125 number of sets of homologous sequences in $M$. floridensis, $M$. incognita and $M$. hapla, and use 126 both gene copy number distributions and gene phylogenies to test the predictions of the different 127 scenarios outlined in Figure 2. 


\section{Materials and Methods}

\section{Nematode materials}

130 DNA from female egg mass cultures of Meloidogyne floridensis isolate 5 was generously

131 sourced and provided from culture by Dr Tom Powers (University of Lincoln, Nebraska, USA)

132 and Dr Janete Brito (Florida Department of Agriculture and Consumer Services, Gainesville, 133 USA).

\section{Sequencing and draft genome assembly}

135 Meloidogyne floridensis DNA was prepared for sequencing using standard Illumina protocols by 136 the GenePool Genomics Facility of the University of Edinburgh. A 260 bp insert library was

137 sequenced using one lane of an Illumina HiSeq2000 (v2 reagents) with 101 base paired-end 138 sequencing. 14.5 gigabases $(\mathrm{Gb})$ of raw sequence data were adapter trimmed and quality 139 filtered using perl and bash scripts to yield $70.2 \mathrm{M}$ pairs totaling $13.2 \mathrm{~Gb}$.

140 The genomic DNA sample derived from nematodes isolated from plant roots, and surrounded, 141 therefore, by the bacterial communities of the rhizosphere. Egg masses of RKN are known to be 142 associated with microbial taxa. To identify potential contaminants, we performed a preliminary 143 assembly of all the trimmed reads ignoring pairing information. We then estimated read 144 coverage of each assembled contig by mapping all reads back to the assembly, and annotated 14510,000 randomly sampled contigs with the taxonomic order of their best megablast (BLAST+ 146 version 2.2.25+ (Zhang et al. 2000)) match to the NCBI nt database (Benson et al. 2011). A 147 taxon-annotated scatter plot of the GC\% and coverage of each contig was used to visualize the 148 contaminants present in the data (Supplementary Figure S1) (Kumar \& Blaxter 2012). Distinct 149 GC\%-coverage clusters in this plot were annotated with distinct taxonomic matches. A major 150 cluster annotated as nematode was clearly dominant. Additional minor clusters were annotated 151 as deriving from the bacterial orders Bacillales, Burkholderiales, Pseudomonadales and 
152 Rhizobiales. These all either had much lower coverage or much higher GC content than the

153 nematode cluster. We conservatively removed contigs that matched the GC content and

154 coverage of the identified contaminant blobs. To ensure optimal contamination removal, a

155 second round of megablast searches was performed and any contigs that matched Bacterial

156 databases were removed. Only reads mapping to the remaining, putatively nematode contigs

157 and their pairs were retained for the next step. The true insert size distribution of these reads

158 was also estimated by mapping the pairs back to the preliminary assembly.

159 A stringent reassembly of the cleaned read set $(11.1 \mathrm{~Gb})$ was performed using reliable coverage

160 information estimated from the preliminary assembly GC\%-coverage plot. Velvet v1.1.04

161 (Zerbino 2010; Zerbino \& Birney 2008) was used with a k-mer value of 55 and the parameters

162 -exp_cov 45, -cov_cutoff 4.5, and -ins_length 260. Other parameters and assemblers were also

163 tried but this assembly had the best contig length optimality scores (e.g. N50, the contig length

164 at which $50 \%$ of the assembly is in contigs of that length or greater) and the highest CEGMA

165 values (using CEGMA version 2.3, (Parra et al. 2007)). Redundant contigs likely to derive from

166 independent assembly of allelic copies were removed using CD-HIT-EST (version 4.5.5, (Li \&

167 Godzik 2006)) with -c 0.97 (removing all contigs that were more than $97 \%$ identical over their

168 entire length to another, longer contig).

\section{Protein predictions and comparisons}

170 A full annotation of the $M$. floridensis draft genome was not carried out, because no

171 transcriptome data for the species was available. Instead, because we were interested in

172 comparing coding sequences conserved with $M$. hapla and $M$. incognita, we used the

173 protein2genome model in exonerate v2.2.0, (Slater \& Birney 2005) to align all M. hapla and $M$.

174 incognita proteins, derived from the published genome sequences, to the $M$. floridensis draft

175 genome. We extracted coding sequences (CDSs) that aligned to at least $50 \%$ of the length of

176 the query protein sequences. If multiple $M$. hapla or $M$. incognita query protein sequences

177 aligned to overlapping loci on the $M$. floridensis genome, only the longest locus was chosen as a 
178 putative $M$. floridensis CDS. The CDSs for all three species were trimmed after the first stop

179 codon, and only sequences with a minimum of 50 amino acids were retained for further analysis.

180 To assess the level of self-identity among CDSs in each species, a BLASTn (version 2.2.25+, 181 (Altschul et al. 1990)) search (with a sensitive E-value cutoff of 1e-5) was performed and the top 182 scoring hit for each sequence to a CDS (other than itself) was selected if the length of the 183 alignment was longer than $70 \%$ of the query sequence. The transcriipts of $M$. incognita were compared to the genomes of $M$. floridensis and $M$. hapla to identify levels of between species similarity using the same strategy.

\section{Clustering}

We used Inparanoid (version 4.1, (Ostlund et al. 2010)) and QuickParanoid (Kim n.d.) with default settings to assign proteins from the three Meloidogyne species to orthology groups. While assessing the level of duplication within the CDS sets (Figure 3), we noted that several $M$. incognita CDS sequences were identical or nearly identical (>98\% identity). These are most likely derived from allelic variants rather than gene duplications (which show a separate peak between 95 and $97 \%$ identity). To simplify the construction of orthologous gene clusters, we reduced these near identical sequences in each species using CD-HIT-EST, removing any CDSs that were at least $98 \%$ identical across their whole length to another CDS.

\section{Phylogenetic analyses}

For each InParanoid cluster, Clustal Omega v1.0.3, (Sievers et al. 2011) was first used to align the protein sequences. Tranalign (from the Emboss suite, v6.2.0, (Rice et al. 2000)) was then used along with the protein alignment as a guide to align the nucleotide CDS sequences. Finally, RAxML v7.2.8, (Stamatakis 2006) was used to create maximum likelihood trees for each set of aligned CDS sequences in three steps: (i) finding the best ML tree by running the GTRGAMMA

201 model for 10 runs using the command "raxmIHPC-PTHREADS-SSE3 -m GTRGAMMA -s \$a -\#

20210 -n \$a -T 2"; (ii) getting the bootstrap support values for this tree by running the same model 
203 until the autoMRE convergence criterion was satisfied employed the command "raxmlHPC204 PTHREADS-SSE3 -m GTRGAMMA -s \$a -\# autoMRE -n \$a.b -T 2 -b 12345"; (iii) using the 205 bootstrap trees to draw bipartitions on the best ML tree used the command "raxmlHPC206 PTHREADS-SSE3 -m GTRCAT -f b -t RAxML_bestTree.\$a -z RAxML_bootstrap.\$a.b -n \$a.I -T 2072 -o mh". Gene trees with a BP support of $70 \%$ or more were included in the analysis. The 208 resulting trees were imported into the R Ape package v2.8, (Paradis et al. 2004) to count the 209 number of trees with the same topology. Datafiles, treefiles, and scripts for processing the trees 210 and other data can be obtained from FigShare doi:10.6084/m9.figshare.978784. 


\section{Results}

\section{The genome of Meloidogyne floridensis}

213 The M. floridensis genome was assembled using $11.1 \mathrm{~Gb}$ of cleaned data (see Supplementary

214 Figure S1) from $116 \mathrm{M}$ reads (an estimated $\sim 100 \mathrm{X}$ coverage), using Illumina HiSeq2000 100

215 base paired-end sequencing of 250 bp fragments. The raw read data have been submitted to 216 the Short Read Archive as accession ERP001338, the final assembly file is available at EMBL, 217 and a blast database, CDS download, and other resources are available at 218 http://brock.bio.ed.ac.uk/M_floridensis/ and

219 http://nematodes.org/genomes/meloidogyne_floridensis

220 Intra-genomic comparisons reveal high numbers of duplicate genes in $M$. incognita and $M$. floridensis

222 Analysis of the distribution of within-genome CDS matches (Figure 3A) identified an unexpected 223 excess of apparent duplication in M. floridensis. While the CDS set of $M$. hapla had a relatively 224 low rate of duplication, and no excess of duplicates of any particular divergence level, both $M$. 225 incognita and $M$. floridensis had many more duplicates and a peak of divergence between 226 duplicates at 95 to $97 \%$ identity. $M$. incognita showed an additional peak at $\sim 100 \%$ identity likely 227 due to a failure to collapse allelic copies of some genes by the original authors (Abad et al. 228 2008). Because of the way we constructed our draft genome assembly, collapsing high-identity 229 assembly fragments before analysis, $M$. floridensis lacked a near complete identity peak. To test 230 for divergent copies of mtDNA within $M$. floridensis we searched the genomic contigs with a $M$. 231 floridensis $16 \mathrm{~S}$ mitochondrial rRNA gene from the international sequence databases (Genbank 232 accession: AY635609.1) using blastn. Different regions of this query matched to two contigs 
comprising 1087 nucleotides in total and we observed a divergence between query and contig of

$2344 / 1087$ nucleotides or $0.37 \%$.

235 The very high frequency of intragenomic duplicate copies with a consistent divergence level

236 strongly suggest that either $M$. floridensis, like $M$. incognita, is a hybrid species, with

237 contributions from two distinct parental genomes, or that it has undergone a whole genome

238 duplication. These distinct possibilities are addressed below. Comparing CDS between species

239 we identified a high frequency of near-100\% identity between $M$. incognita and its best match in

240 the $M$. floridensis genome (Figure 3B). This pattern was not evident when $M$. incognita was

241 compared to M. hapla.

\section{Distinguishing sibling from parent-child species relationships}

243 We identified several models that might explain the observed levels of within-genome divergent

244 duplicates in $M$. incognita and $M$. floridensis (Figure 3A). Expectations of relative numbers of 245 (homeologous) gene copies per species, and the phylogenetic relationships of these 246 homeologue sets differ and allow us to distinguish between the models. Thus for example under 247 scenario 3 (Figure 2B) we test to determine if $M$. incognita has two divergent homeologous gene 248 copies, one of which is phylogenetically very closely related to the (collapsed) allelic copies in $M$. 249 floridensis. We therefore clustered the CDS of the three species using InParanoid, after 250 removing all CDS encoding peptides less than 50 amino acids in length.

251 We defined 11,587 clusters that contained CDS from more than one species, and 4,018 that had 252 representatives from all three species (Supplementary Figure S2). These represent a number 253 and proportion similar to comparisons between other nematode species with complete genomes 254 (e.g. 2,501 clusters were previously identified containing representatives from four nematode 255 genomes (Mitreva et al. 2011)). As M. hapla is not expected to have undergone whole genome 256 duplication, and we find no evidence of an excess of diverged duplicates in the $M$. hapla 257 genome, we selected homologous gene sets where the ancestral gene was likely to have been 258 single-copy by excluding clusters with more than one $M$. hapla member, and those lacking $M$. 
hapla members. We classified these clusters by the numbers of $M$. incognita and $M$. floridensis

260 genes they contained (Table 2; Figure 4). The trees generated and the scripts used to parse

261 them into the categories represented in Figure 4 are available through FigShare

262 doi:10.6084/m9.figshare.978784.

263 The process of idiosyncratic gene loss (or failure to capture a gene in the draft sequencing and 264 assembly) is evident in the numbers of genes that have one $M$. hapla representative and no 265 members from either $M$. incognita (column 1 of Table 2) or $M$. floridensis (row 1 of table 2). Here 266 it is striking that the clusters that contain only one M. hapla and one $M$. floridensis member 267 (Mh1:Mf1:Mi0) outnumber by approximately two to one clusters that have one M. hapla and one 268 M. incognita member (Mh1:Mf0:Mi1). This suggests that the $M$. floridensis genome draft is a 269 good substrate for these analyses (it contains homologues of many conserved genes apparently 270 lost from, or missing in the draft assembly of, the $M$. incognita genome), and that the $M$. 271 incognita draft is either incomplete or has experienced greater rates of gene loss.

272 The numbers of genes present in clusters that have two or more members, but lack one of $M$. 273 floridensis or $M$. incognita (for example the 226 Mh1:Mf2:Mi0 clusters) reveal the potential extent 274 of within-lineage duplication and divergence (and a component of stochastic loss of several 275 homeologues in the missing species). There is no excess of these classes of cluster in $M$. 276 incognita, arguing against a within-lineage, whole-genome duplication (i.e. against scenarios 1 277 or 2; Figure 2A).

278 The striking feature of the membership of clusters (Table 2) is the number of cases where $M$. 279 incognita has more cluster members than does $M$. floridensis. Thus there are 920 clusters in the 280 class Mh1:Mf1:Mi2, but only 257 in the class Mh1:Mf2:Mi1, and 102 clusters in the class 281 Mh1:Mf1:Mi3 compared to 17 in the class Mh1:Mf3:Mi1. This finding argues for the presence in $282 M$. incognita of at least one more genome copy than in $M$. floridensis, i.e. that $M$. incognita is 283 likely to be a degenerate triploid hybrid (scenario 5, Figure 2D). It is possible that some of the 
284 clusters in the Mh1:Mf1:Mi0 and Mh1:Mf0:Mi1 sets arise from M. floridensis and M. incognita 285 being derived from different, divergent parents.

286

287

288

289

290

291

292

293

294

295

296

297

298

299

300

301

302

303

\section{Phylogenomic analysis of homologue relationships}

A second set of predictions from the models in Figure 2 concerns the phylogenetic relationships of the resulting sets of homologous gene sequences. Each model predicts a particular set of relationships between gene copies in each species. We therefore analyzed each informative set of clusters represented in Table 2 to identify which alternate topology was supported, assuming in each case that the single $M$. hapla representative was the outgroup. These phylogenomic results are summarized in Figure 4. For each informative set of clusters, the majority topology supported scenario 5 (Figure 2D), i.e. that M. floridensis is a hybrid, and was one of the parent species in a hybridization event that gave rise to a triploid $M$. incognita. Thus for the 902 Mh1:Mf1:Mi2 clusters, the topology in which one M. incognita CDS groups with the M. floridensis CDS to the exclusion of the other $M$. incognita sequence was favoured in $79 \%$ of the clusters, while in only 201 clusters (21\%) the two M. incognita genes instead appeared to have arisen by duplication within $M$. incognita. In the Mh1:Mf2:Mi2 cluster set, one third of the clusters supported the topology where there were two independent sister relationships between $M$. incognita and $M$. floridensis genes. A further $48 \%$ of the trees were congruent with a triploid status for $M$. incognita where gene loss (or lack of prediction) had removed one $M$. incognita representative. The other classes of clusters could be interpreted in the same manner, and displayed trends that supported scenario 5. 


\section{Discussion}

305 The genome structure and content of tropical Meloidogyne is revealed by our analyses to have

306 had complex origins. It is likely that hybridization, ploidy change, and subsequent aneuploidy

307 have all played a role in the evolution of the diversity in this genus. The molecular evolutionary

308 patterns revealed by comparative genomics however give us tools to conduct detailed analysis

309 of these histories. This approach allows us to interpret the evolution of different reproductive 310 strategies in terms of genome change, and better understand the evolution of these 311 polyphagous pathogens.

\section{The M. floridensis genome reveals its hybrid origins}

313 Our draft assembly of the genome of $M$. floridensis reveals a relatively typical nematode

314 genome. The base haploid genome size for Meloidogyninae is likely to be $\sim 50 \mathrm{Mb}$. Both the 315 sequenced genome of $M$. hapla (Opperman et al. 2008), and independent measurement of its 316 genome size from densitometry (Pableo \& Triantaphyllou 1989), yield estimates of 50-54 Mb.

317 The sequenced genome estimate is unlikely to be inflated through issues of uncollapsed haploid 318 contigs, as $M$. hapla is expected to have reduced heterozygosity through its automictic 319 reproductive mode (Liu et al. 2007), and the sequenced strain was inbred (Opperman et al. 320 2008). Hybrid taxa, containing homeologous chromosomes from more than one parental 321 lineage, would be expected to have genome assembly sizes that are the sum of the parental 322 genomes, albeit modified by idiosyncratic post-hybridization gene loss and repeat copy change. 323 Thus the $\sim 100 \mathrm{Mb}$ genome size estimated for $M$. floridensis is in keeping with a base 324 Meloidogyninae genome of $\sim 50 \mathrm{Mb}$, with homeologous sequences assembled independently.

325 The divergence between inferred homeologous genes in our genome ( 4-8\%) would preclude 326 coassembly of homeologous coding sequences, and the higher divergence found in intergenic 327 and intronic sequences would make them even less likely to be coassembled. The published $M$. 328 incognita genome is $86 \mathrm{Mb}$, but ongoing revision of the assembly suggests a true value of $\sim 130$ 
$329 \mathrm{Mb} \mathrm{Mb}$ (Etienne Danchin, personal communication), as might be expected for a hypo-triploid 330 species.

331 The $M$. floridensis genome assembly is less contiguous than those of $M$. hapla and M. incognita 332 (reflected in the lower contiguity and content of conserved eukaryotic genes). Such 333 fragmentation is a known limitation of using a single small-insert paired-end library, and 334 refinement of the assembly using larger-insert mate pair, or long single molecule reads, would 335 undoubtedly improve the biological completeness of the product. Our primary aim however was not to produce a highly contiguous assembly, but rather to identify protein-coding sequences (CDS) for use in comparative genomic analyses. Despite the fragmentation we were able to identify over 15,000 CDS segments to address the possible hybrid status of $M$. floridensis and M. incognita, making it more than sufficient for this study.

We note that both the $M$. incognita and the $M$. floridensis genomes have low scores $(60-75 \%)$ when assessed by the Core Eukaryotic Genes Mapping Approach (CEGMA), compared to the $94 \%$ scored by the $M$. hapla assembly (and assemblies of other nematode genomes). However, the published $M$. incognita genome, while having much better assembly statistics (only 9,538 scaffolds, and a contiguity $\sim 4$ times that achieved for $M$. floridensis), has similarly poor scores in

345 CEGMA analysis. Whether this is a reflection of shared divergent biology, or, as we suspect, a 346 poor fragmented assembly, will require additional sequencing data, reassembly and 347 reassessment.

348 The phylogenetic position of the automictic $M$. floridensis suggest that this species, or an 349 immediate ancestor, was parental to the tropical apomicts, i.e. being one partner in the hybrid 350 origins of the group (scenarios 3 and 5 , Figure $2 \mathrm{~B}, \mathrm{D}$ ). It is also possible however that $M$. 351 floridensis is not directly parental to the apomicts, but rather a hybrid sibling, also arising by 352 interspecific hybridization (scenario 4, Figure 2C). In this case one parent of $M$. floridensis is 353 very likely to also have been involved in the hybrid origins of $M$. incognita as very many loci were 354 found to be nearly identical between $M$. incognita and $M$. floridensis (Figure 3B). In order to 
355 distinguish between scenario 3 (diploid parent), scenario 4 (hybrid sibling) and scenario 5 (hybrid

356 parent) we examined the sequence diversity within each species' genome.

\section{Intra-genomic Divergence of Coding Loci}

358 Information concerning the hybrid status of $M$. floridensis can be gained from comparing the

359 pattern of gene duplication within its genome to that of other RKN species, since Meloidogyne

360 incognita has been suggested previously to have hybrid origins (Dalmasso \& Berge 1983;

361 Triantaphyllou 1985; Hugall et al. 1999; Castagnone-Sereno 2006; Lunt 2008) whereas M. hapla

362 never has. An interspecific hybrid would be expected to have an excess of divergent intra363 genomic duplicates compared to a non-hybrid, due to its homeologous chromosome pairs. The 364 genome of $M$. hapla, a closely related species without a hybrid origin, represents the normal 365 intra-genomic duplication pattern without homeologous chromosomes. In M. hapla there was a 366 very much lower number of divergent duplicates compared to the other species, and these had a 367 wide range of divergences rather than a frequency peak at any divergence value. While there 368 was a slight excess of duplicates with high identity in $M$. hapla, the distribution overall is 369 consistent with an ongoing rare process of stochastic duplication followed by gradual divergence 370 (Figure 3A).

371 In contrast to the pattern observed in $M$. hapla, the intra-genomic comparisons of both $M$. 372 incognita and $M$. floridensis revealed many more divergent duplicated CDS (Figure 3A). We 373 observed a peak of high-identity duplicates in $M$. incognita that was absent in $M$. floridensis. This 374 is most likely because we stringently collapsed high identity segments (as putative allelic copies) 375 during assembly of $M$. floridensis whereas the $M$. incognita genome assembly may still contain 376 some of these alleles. Most striking however was the presence in both species of a frequency 377 peak of more diverged duplicates showing $\sim 96 \%$ identity. Such duplicates have been described 378 in M. incognita (Abad et al. 2008; Lunt 2008) although the scale of these diverged loci and their 379 presence in $M$. floridensis has not been reported previously. 
380 If the M. floridensis divergent copies were the product of a mixed sample, and thus represented 381 polymorphism rather than homeologs, we would predict that mitochondrial DNA would also 382 display this pattern. Our BLAST search however did not find any divergent contigs matching to 383 our mtDNA genbank query. The query sequence differed by only $0.37 \%$ and this divergence 384 between the genbank sequence and our strain is typical of intraspecific polymorphism levels in 385 other nematodes but is approximately ten-fold less than we observe between the nuclear 386 divergent copies in the M. floridensis genome. We therefore do not consider that, even if our 387 starting material had been contaminated with a second $M$. floridensis strain, such intraspecific 388 polymorphism could account for our much more diverged genomic copies.

Ongoing individual gene duplication events - which we propose has generated the M. hapla distribution - could not have produced these patterns. Instead, the distributions are congruent with either a single major past event of genome duplication followed by divergence, or else hybridization to bring together pre-diverged homeologous chromosome copies that had been evolving independently since the last common ancestor of the parental species. On top of these processes differences in the rates of evolution of individual loci has resulted in variation in observed identity in the extant genomes, producing a distribution around a single peak of 396 divergence. While these two alternative scenarios (endoduplication and homeologous 397 chromosomes) cannot be distinguished on the basis of duplicate divergence data alone, the analysis does suggest that the genome content of both $M$. floridensis and $M$. incognita have been shaped in very similar ways by major duplication or divergence events.

401 To distinguish between endoduplication and hybrid origins of these CDS divergences, we 402 examined the phylogenetic histories of sets of homologous loci from the three Meloidogyne 403 genomes. By selecting CDS clusters with only a single member from the $M$. hapla genome we 404 have likely restricted our analyses to loci that were single copy in the last common ancestor of 405 the three species, and thus do not show the complexities of turnover in large multigene families. 
406 We compared support on a gene-by-gene basis for tree topologies that would support or refute 407 the hybrid versus endoduplication scenarios (Figure 2, Table 2 and Figure 4). Using this 408 approach we could robustly exclude scenario 1, endoduplication of the $M$. incognita genome, as 409 a source of duplicate CDS since we frequently observed that these $M$. incognita sequences 410 were not monophyletic with respect to $M$. floridensis. If $M$. incognita had duplicated its own 411 genome we would expect these duplicate CDS to share a recent origin and be each other's 412 closest relatives. We could similarly exclude scenarios 2 and 3 , since intra-genomic 413 comparisons of CDS in the $M$. floridensis genome revealed that it also possesses divergent 414 duplicates, and phylogenetic analyses indicated that these, just like the $M$. incognita sequences, 415 are not monophyletic by species.

416 Thus we suggest that the most parsimonious explanation of the duplicate divergence and 417 phylogenetic data is that both $M$. floridensis and $M$. incognita are hybrid species, and the 418 duplicate CDS are homeologues rather than within-species paralogues. We can distinguish 419 between scenario 4 (independent hybrid origins: the two species are step-sisters) and scenario 5 420 (M. floridensis represents one of the parents of a triploid hybrid $M$. incognita) by phylogenetic 421 analyses of the clustered CDS. We observed an excess of clusters where there were more $M$. 422 incognita members than there were $M$. floridensis members, as would be expected from a 423 triploid species, whether or not it was now losing duplicated genes stochastically. In these 424 clusters, the extra $M$. incognita CDS was less likely to be sister to one of the other $M$. incognita 425 CDS than it was to be a sister to a $M$. incognita - $M$. floridensis pair. Based on these data we 426 suggest that the triplicate loci in $M$. incognita are the three homeologues that have resulted from 427 a hybridization event between the hybrid $M$. floridensis and an unidentified second, likely non428 hybrid, parent (scenario 5, Figure 2D). For clusters containing two M. floridensis homeologues 429 and two M. incognita homeologues, the topology supporting shared hybrid ancestry was again 430 more frequently recovered than topologies supporting independent hybridization events. 
431 Handoo et al (2004) described the meiosis of $M$. floridensis as lacking a second maturation

432 division and being 'intermediate' between meiotic and mitotic forms of reproduction. The division

433 observed by Handoo et al (2004) is in fact likely a long-known form of purely meiotic automixis

434 called "first division restitution". Bell (1982, p.40), in his classic review of the evolution of mating

435 systems, describes one of the three primary types of automixis as involving the suppression of

436 the second meiotic division, exactly as described by Handoo et al (2004) for M. floridensis. The

437 maintenance of both homeologs in the M. floridensis genome through meiotic divisions, as we

438 report here, may seem more challenging than in $M$. incognita, which reproduces only by mitosis.

439 Automixis that maintains the parental heterozygosity is however well described in other animals

440 and we assume a very similar mechanism occurs in M. floridensis (Bell 1982, p.40; Smith 1978,

441 p.44; Hood \& Antonovics 2004 and refs therein).

\section{Hybrid speciation and adaptive novelty}

443 Animal hybrids have been characterized as rare, unfit, and adversely affected by both 444 competition and gene flow from their parents (Mayr 1963; Barton 2001). There is now an 445 increasing awareness in the literature however of animal hybridization as both a speciation 446 mechanism and a route to the generation of novel phenotypic diversity on which natural 447 selection may act (Bullini 1994; Arnold 1997; Mavarez \& Linares 2008; P. S. Soltis \& D. E. Soltis 448 2009; Abbott et al. 2013). There are a growing number of cases in which animal species have a 449 hybrid origin, i.e.: it is known that all vertebrate constitutive parthenogens, and gynogenetic 450 species have hybrid origins (Avise 2008); the Italian sparrow (Passer italiae) has been shown to 451 be a nascent hybrid species (Hermansen et al. 2011); hybridization between two species of 452 Rhagoletis tephritid fruitflies has led to expansion into a novel ecological niche (host plant) in the 453 hybrid, and also reproductive isolation from both parents since mating is confined to the host 454 plant (Schwarz et al. 2005). The genetic basis of hybridization in generating adaptive diversity 455 has been revealed in a number of studies: the Heliconius melpomene genome demonstrates 456 that hybridization and introgression has been important for the adaptive radiation of these 
butterflies, by sharing protective colour-pattern genes among co-mimics (Dasmahapatra et al. 458 2012); the Northern European freshwater 'invasive sculpin' fish are hybrids between two 459 geographically isolated Cottus species and they have colonized a novel niche consisting of the 460 extensively human-altered lower reaches of the rivers Rhine and Scheldt (Czypionka et al. 461 2012); The cichlid adaptive radiation in Lake Malawi involves the evolution of more than 400 462 species, over a period of only 4.6 million years (Genner et al. 2007), which have colonized and 463 adapted to many diverse lacustrine habitats. Recent genetic studies indicate that this radiation, 464 and cichlid diversification in general, has been strongly influenced by interspecific hybridization 465 (Joyce et al. 2011; Schwarzer et al. 2012; Loh et al. 2013; Genner \& Turner 2012).

466 It has been suggested that hybrid animal taxa are most likely to succeed where new habitats 467 open up, and such events may have played a significant role in several classic examples of 468 adaptive radiation (Seehausen 2006; Abbott et al. 2013; Seehausen 2013; Kearney 2005). The 469 tropical RKN are exceptionally successful globally-distributed pathogens of diverse agricultural 470 crops (Moens et al. 2009; Trudgill \& Blok 2001). These species have colonized a novel habitat, 471 show extensive functional diversity, and have adapted to crop host-plants in the very brief 472 evolutionary timeframe that agriculture has existed (a few thousand years). This is a situation 473 similar to other animal adaptive radiations where hybridization may also have played a 474 significant role (Seehausen 2006; Seehausen 2013; Abbott et al. 2013).

475 Although the adaptive consequences of hybridization are being increasingly recognized as 476 important for biodiversity, ecology and evolution, the origin of novel traits, colonization of new 477 ecological niches, and adaptive evolution can lead to serious problems if the organisms 478 concerned are pathogens of humans, livestock, or crops (Bisharat et al. 2005; Brasier 2001; 479 Stukenbrock et al. 2012; Inderbitzin et al. 2011; Goss et al. 2011). It is particularly important 480 therefore to understand the genetic basis of adaptive diversification in relation to existing or 481 emerging pathogens. 
482 The tropical apomictic RKN, exemplified by M. incognita, M. arenaria and M. javanica, possess

483 host ranges that may include practically all agriculturally important species overlapping their

484 distribution, causing $M$. incognita to be described as the "single most damaging crop pathogen in

485 the world" (Trudgill \& Blok 2001). Such extreme polyphagy is not typically encountered in

486 Meloidogyne species outside of the radiation of tropical apomicts, although some do exploit

487 multiple hosts. The origins and mechanisms of this greatly expanded host range are not only

488 interesting from an evolutionary genomics perspective but also important to our understanding of

489 the mode of action of these globally important crop pathogens. The demonstration of the hybrid

490 origins of $M$. incognita and $M$. floridensis, and by implication $M$. javanica and $M$. arenaria also,

491 suggests transgressive segregation of adaptive variation might have played an important role in

492 determining host range. Transgressive segregation is when the absolute values of traits in some

493 hybrids exceed the trait variation shown by either parental lineage. Such transgressive

494 phenotypes are common in hybrid offspring in both animals and plants, and particularly so where

495 the parents derive from inbred but divergent lineages (Rieseberg, Archer, et al. 1999a).

496 Transgressive phenotypes have played a significant role in plant breeding, where crossing of

497 inbred parental lineages can lead to extreme offspring variation onto which artificial selection is

498 imposed, and similar processes are likely to act on hybrid swarms resulting from natural

499 selection acting on inter-species crosses in the wild (Rieseberg, Archer, et al. 1999a; Stelkens \&

500 Seehausen 2009; Genner \& Turner 2012). We do not yet know whether transgressive

501 phenotypes in hybrid apomict RKN have been shaped by natural selection, but given our

502 increasing awareness of its importance in adaptive radiations, and the frequency with which

503 hybrid plant pathogens are detected in other systems (Stukenbrock et al. 2012; Stukenbrock \&

504 McDonald 2008; Inderbitzin et al. 2011; Brasier 2001), it may be an important direction for future

505 research allowing us to detect likely pathogens at early stages.

506 Although we have not yet identified the parental taxa of $M$. floridensis, or the second parent of

507 the tropical apomict RKN, it is likely that they were facultatively sexual meiotic parthenogens, as

508 this is the most common reproductive mode within Meloidogyne (Triantaphyllou 1982; 
509 Triantaphyllou 1985; Chitwood \& Perry 2009). This breeding system can fuse the products of a

510 single meiotic division in order to regain diploidy, making these taxa more similar to the inbred

511 lineages of plants highlighted as frequent sources of transgressive segregation and extreme

512 phenotypes (Rieseberg, Whitton, et al. 1999b) than to the typical (amphimictic) species of

513 hybridizing animals. If this "polyphagy as transgressive segregation" hypothesis were correct

514 then we would predict that the parents of the polyphagous RKN would most likely be automicts

515 with considerably smaller host ranges.

\section{Hybridization and molecular genetic approaches to Meloidogyne diversity}

517 Molecular approaches to understanding the diversity of apomictic RKN have a long history and

518 include studies of isozymes, mitochondrial DNA (mtDNA), ribosomal internal transcribed spacer

519 (ITS), ribosomal RNA genes (rDNA), random amplified polymorphic DNA markers (RAPDs),

520 amplified fragment length polymorphisms (AFLPs), and other marker systems (see Blok and

521 Powers (2009) for a review). However, if some Meloidogyne species are in fact hybrids, this 522 presents particular problems for the standard molecular approaches used to characterize

523 diversity. These typically assume that species or isolates have diverged following a bifurcating,

524 tree-like, evolutionary pathway. Hybridization violates this assumption and produces more 525 complex evolutionary histories that can either be misrepresented by single locus markers, or 526 else produce intermediate or equivocal signal from multi-locus approaches. For example, a

527 major reason that mtDNA and rDNA sequencing have been useful in evolutionary ecology is that 528 they are effectively haploid, and hybrid taxa, which often retain just one of their parental species' 529 genotypes at these loci, present particular problems for these approaches (Seehausen 2006; 530 Hailer et al. 2012; Meyer et al. 2012). While carefully benchmarked marker approaches may still 531 have utility in diagnostics, they will not be able to accurately reflect the complex evolutionary 532 pathway of hybrid Meloidogyne species where different loci are likely to have experienced very 533 different histories. Incongruence between markers is therefore to be expected as a true 534 reflection of history, rather than due to a lack of analytical power. We are currently in the early 
535 stages of Meloidogyne comparative genomics and current estimates of the complex

536 phylogenetic relationships between hybrid taxa will need to be constantly refined as more 537 species are added.

538 Genomic approaches to the RKN system hold many advantages, including documenting the

539 genomic changes associated with host-specialization, extreme polyphagy, and interaction with 540 plant defense systems. An interesting and important question now is whether the main apomictic 541 RKN species have a single origin, with species divergence perhaps related to aneuploidy, or are 542 instead the result of repeated hybridizations of the same or similar parental lineages. Different 543 patterns of origin may determine the extent to which control strategies may be broadly or only 544 locally applicable. Finally, if transgressive segregation is a cause of extreme and unique 545 diversity, including polyphagy and novel resistance breaking isolates, then monitoring of new 546 hybrid lineages may be an agricultural necessity. We are now close to the time where RKN 547 isolates can be characterized not only with a trivial name (e.g. M. incognita race X) but instead a 548 detailed list of genome wide variants and their known association with the environment, 549 response to nematicides, and virulence against a range of plant host species and genotypes 550 an approach that will surely be extremely valuable in optimizing agricultural success. We caution 551 therefore that although traditional genetic approaches may be valuable for rapid diagnostics, 552 population genomics must be embraced in order to really advance our understanding of these 553 important pathogens and maximize our ability to successfully intervene.

\section{Conclusions}

555 Here we have used whole genome sequencing and evolutionary comparative genomics to 556 demonstrate the complex hybrid origins of key Root Knot Nematode species. Understanding the 557 evolutionary history of Meloidogyne species is a priority since only by this route can the evolution 558 of pathogenicity and resistance, the emergence of new pathogenic strains, horizontal transfer of 559 genes, and geographic spread of one of the world's most important crop pathogens be properly 560 understood. The importance of animal hybridization to speciation and adaptation is being 
561 increasingly recognized, driven by new insights from genome sequencing. Meloidogyne 562 incognita is shown to be an unusual double-hybrid, suggesting that hybridization may be a 563 common and complex process in the history of this group. The Meloidogyne system, with its very 564 recent expansion to fill numerous agricultural ecological niches, shows interesting parallels to 565 natural adaptive radiations that may also have been greatly influenced by hybridization. Further 566 work elucidating whether hybridization contributes adaptively to polyphagy will be important not 567 just in the context of root knot nematodes, but also in determining the interplay of evolutionary 568 forces generating organismal adaptive divergence more generally. 


\section{Acknowledgements}

570 We thank Tom Powers and Janete Brito for sourcing and supplying M. floridensis materials,

571 Etienne Danchin for access to $M$. incognita genome data and Marian Thomson and members of

572 the GenePool Genomics Facility for sequencing support. We thank Africa Gómez, Amir

573 Szitenberg, Steve Moss, Richard Ennos and Karim Gharbi for comments on the manuscript and

574 the project. 
576

577

578

579

580

581

582

583

584

585

586

587

588

589

590

591

592

593

594

595

596

597

Abad, P. et al., 2008. Genome sequence of the metazoan plant-parasitic nematode Meloidogyne incognita. Nature biotechnology, 26(8), pp.909-915. Available at: http://www.ncbi.nlm.nih.gov/pubmed/18660804.

Abbott, R. et al., 2013. Hybridization and speciation. Journal of Evolutionary Biology, 26(2), pp.229-246.

Altschul, S.F. et al., 1990. Basic local alignment search tool. Journal of molecular biology, 215(3), pp.403-410.

Arnold, M.L., 1997. Natural Hybridization and Evolution, New York: Oxford University Press.

Avise, J., 2008. Clonality:The Genetics, Ecology, and Evolution of Sexual Abstinence in Vertebrate Animals, Oxford University Press, USA.

Barton, N.H., 2001. The role of hybridization in evolution. Molecular Ecology, 10(3), pp.551-568.

Bell, G., 1982. The Masterpiece of Nature: The Evolution and Genetics of Sexuality, University of California Press ISBN 0520045831

Benson, D.A. et al., 2011. GenBank. Nucleic Acids Research, 39(Database issue), pp.D32-7.

Bisharat, N. et al., 2005. Hybrid Vibrio vulnificus. Emerging infectious diseases, 11(1), pp.30-35.

Blok, V.C. \& Powers, T.O., 2009. Biochemical and molecular identification. In Root-knot nematodes. Wallingford, UK: CABI Publishing, pp. 98-118.

Brasier, C.M., 2001. Rapid evolution of introduced plant pathogens via interspecific hybridization. Bioscience, 51(2), p.123.

Bullini, L., 1994. Origin and evolution of animal hybrid species. Trends In Ecology \& Evolution, 9(11), pp.422-426.

Castagnone-Sereno, P., 2006. Genetic variability and adaptive evolution in parthenogenetic rootknot nematodes. Heredity, 96(4), pp.282-289.

Chitwood, D.J. \& Perry, R.N., 2009. Reproduction, Physiology and Biochemistry. In R. N. Perry, M. Moens, \& J. L. Starr, eds. Root-knot nematodes. CAB International, pp. 182-200.

Czypionka, T. et al., 2012. Transcriptome changes after genome-wide admixture in invasive sculpins (Cottus). Molecular Ecology, 21(19), pp.4797-4810.

Dalmasso, A. \& Berge, J.B., 1983. Enzyme polymorphism and the concept of parthenogenetic species, exemplified by Meloidogyne. In S. AR, P. HM, \& K. LF, eds. Concepts in nematode systmatics. Academic Press.

Dasmahapatra, K.K. et al., 2012. Butterfly genome reveals promiscuous exchange of mimicry adaptations among species. Nature, 487, pp.94-98.

De Ley, I.T. et al., 2002. Phylogenetic Analyses of Meloidogyne Small Subunit rDNA. Journal of 
Genner, M.J. \& Turner, G.F., 2012. Ancient hybridization and phenotypic novelty within Lake Malawi's cichlid fish radiation. Molecular Biology and Evolution, 29(1), pp.195-206.

612

Genner, M.J. et al., 2007. Age of cichlids: new dates for ancient lake fish radiations. Molecular Biology and Evolution, 24(5), pp.1269-1282.

Goldberg, E.E. \& Igić, B., 2008. On phylogenetic tests of irreversible evolution. Evolution; international journal of organic evolution, 62(11), pp.2727-2741.

Goss, E.M. et al., 2011. The plant pathogen Phytophthora andina emerged via hybridization of an unknown Phytophthora species and the Irish potato famine pathogen, $P$. infestans. PLoS ONE, 6(9), p.e24543.

Hailer, F. et al., 2012. Nuclear Genomic Sequences Reveal that Polar Bears Are an Old and Distinct Bear Lineage. Science (New York, NY), 336(6079), pp.344-347.

Handoo, Z.A. et al., 2004. Morphological, Molecular, and Differential-Host Characterization of Meloidogyne floridensis n. sp. (Nematoda: Meloidogynidae), a Root-Knot Nematode Parasitizing Peach in Florida. Journal of Nematology, 36(1), pp.20-35.

Hermansen, J.S. et al., 2011. Hybrid speciation in sparrows I: phenotypic intermediacy, genetic admixture and barriers to gene flow. Molecular Ecology, 20(18), pp.3812-3822.

Holterman, M. et al., 2009. Small Subunit rDNA-Based Phylogeny of the Tylenchida Sheds Light on Relationships Among Some High-Impact Plant-Parasitic Nematodes and the Evolution of Plant Feeding. Phytopathology, 99(3), pp.227-235.

Hood, M.E. \& Antonovics, J., 2004. Mating within the meiotic tetrad and the maintenance of genomic heterozygosity. Genetics, 166(4), pp.1751-1759.

Hugall, A., Stanton, J. \& Moritz, C., 1999. Reticulate evolution and the origins of ribosomal internal transcribed spacer diversity in apomictic Meloidogyne. Molecular Biology and Evolution, 16(2), pp.157-164.

Inderbitzin, P. et al., 2011. The ascomycete Verticillium longisporum is a hybrid and a plant pathogen with an expanded host range. PLoS ONE, 6(3), p.e18260.

Jeyaprakash, A. et al., 2006. Differentiation of Meloidogyne floridensis from M. arenaria using high-fidelity PCR amplified mitochondrial AT-rich sequences. Nematropica, 36(1), pp.1-12. Available at: http://brokert10.fcla.edu/DLData/NM/NM00000014/NM00995444/1\_12.pdf.

Joyce, D.A. et al., 2011. Repeated colonization and hybridization in Lake Malawi cichlids. Current Biology, 21(6), p.526.

Judson, O.P. \& Normark, B.B., 1996. Ancient asexual scandals. Trends In Ecology \& Evolution, 11(2), pp.41-46.

Kearney, M., 2005. Hybridization, glaciation and geographical parthenogenesis. Trends In Ecology \& Evolution, 20(9), pp.495-502.

Kim, T., QuickParanoid - A Tool for Ortholog Clustering. pl.postech.ac.kr. 
Kumar, S. \& Blaxter, M.L., 2012. Simultaneous genome sequencing of symbionts and their hosts. Symbiosis, 55(3), pp.119-126.

Li, W. \& Godzik, A., 2006. Cd-hit: a fast program for clustering and comparing large sets of protein or nucleotide sequences. Bioinformatics (Oxford, England), 22(13), pp.1658-1659.

Liu, Q.L., Thomas, V.P. \& Williamson, V.M., 2007. Meiotic parthenogenesis in a root-knot nematode results in rapid genomic homozygosity. Genetics, 176(3), pp.1483-1490. Available at: http://www.pubmedcentral.nih.gov/articlerender.fcgi? artid=1931544|\&tool=pmcentrezl\&rendertype=abstract.

Loh, Y.-H.E. et al., 2013. Origins of Shared Genetic Variation in African Cichlids. Molecular Biology and Evolution.

Lunt, D., 2008. Genetic tests of ancient asexuality in root knot nematodes reveal recent hybrid origins. BMC Evolutionary Biology, 8(1), p.194.

Mallet, J., 2007. Hybrid speciation. Nature, 446(7133), pp.279-283.

Mallet, J., 2005. Hybridization as an invasion of the genome. Trends In Ecology \& Evolution, 20(5), pp.229-237.

Mavarez, J. \& Linares, M., 2008. Homoploid hybrid speciation in animals. Molecular Ecology, 17(19), pp.4181-4185.

Mayr, E., 1963. Animal species and evolution, Harvard University Press.

Meyer, M. et al., 2012. A High-Coverage Genome Sequence from an Archaic Denisovan Individual. Science (New York, NY), 338(6104), pp.222-226.

Mitreva, M. et al., 2011. The draft genome of the parasitic nematode Trichinella spiralis. Nature Genetics, 43(3), pp.228-235.

Moens, M., Perry, R.N. \& Starr, J.L., 2009. Meloidogyne species - a Diverse Group of Novel and Important Plant Parasites. In Root-knot nematodes. CAB International, pp. 1-17.

Nolte, A.W. \& Tautz, D., 2010. Understanding the onset of hybrid speciation. Trends in Genetics, 26(2), pp.54-58.

Opperman, C.H. et al., 2008. Sequence and genetic map of Meloidogyne hapla: A compact nematode genome for plant parasitism. Proceedings of the National Academy of Sciences of the United States of America, 105(39), pp.14802-14807.

Ostlund, G. et al., 2010. InParanoid 7: new algorithms and tools for eukaryotic orthology analysis. Nucleic Acids Research, 38(Database issue), pp.D196-203.

Pableo, E.C. \& Triantaphyllou, A.C., 1989. DNA complexity of the root-knot nematode (Meloidgyne spp.) genome. Journal of Nematology, 21, pp.260-263.

Paradis, E., Claude, J. \& Strimmer, K., 2004. APE: Analyses of Phylogenetics and Evolution in R language. Bioinformatics (Oxford, England), 20(2), pp.289-290.

Parra, G., Bradnam, K. \& Korf, I., 2007. CEGMA: a pipeline to accurately annotate core genes in eukaryotic genomes. Bioinformatics (Oxford, England), 23(9), pp.1061-1067. 
Rice, P., Longden, I. \& Bleasby, A., 2000. EMBOSS: the European Molecular Biology Open Software Suite. Trends in Genetics, 16(6), pp.276-277.

Rieseberg, L.H., Archer, M.A. \& Wayne, R.K., 1999a. Transgressive segregation, adaptation and speciation. Heredity, 83 ( Pt 4), pp.363-372.

Rieseberg, L.H., Whitton, J. \& Gardner, K., 1999b. Hybrid zones and the genetic architecture of a barrier to gene flow between two sunflower species. Genetics, 152(2), pp.713-727.

Sasser, J.N. \& Carter, C.C., 1985. Overview of the International Meloidogyne Project 1975-1984. In K. R. Barker, C. C. Carter, \& J. N. Sasser, eds. An Advanced treatise on Meloidogyne. Dept. of Plant Pathology. North Carolina State Univ.

Schwarz, D. et al., 2005. Host shift to an invasive plant triggers rapid animal hybrid speciation. Nature, 436(7050), pp.546-549.

Schwarzer, J. et al., 2012. Repeated trans-watershed hybridization among haplochromine cichlids (Cichlidae) was triggered by Neogene landscape evolution. Proceedings Biological sciences / The Royal Society, 279(1746), pp.4389-4398.

Schwenk, K., Brede, N. \& Streit, B., 2008. Introduction. Extent, processes and evolutionary impact of interspecific hybridization in animals. Philosophical Transactions of the Royal Society B: Biological Sciences, 363(1505), pp.2805-2811.

Seehausen, O., 2013. Conditions when hybridization might predispose populations for adaptive radiation. Journal of Evolutionary Biology, 26(2), pp.279-281.

Seehausen, O., 2006. Hybridization and adaptive radiation. Trends In Ecology \& Evolution, 19(4), pp.198-207.

Sievers, F. et al., 2011. Fast, scalable generation of high-quality protein multiple sequence alignments using Clustal Omega. Molecular systems biology, 7, p.539.

Slater, G.S.C. \& Birney, E., 2005. Automated generation of heuristics for biological sequence comparison. BMC Bioinformatics, 6, p.31.

Smith, J., 1978. The Evolution of Sex, Cambridge University Press.

Soltis, P.S. \& Soltis, D.E., 2009. The role of hybridization in plant speciation. Annual review of plant biology, 60, pp.561-588.

Stamatakis, A., 2006. RAxML-VI-HPC: maximum likelihood-based phylogenetic analyses with thousands of taxa and mixed models. Bioinformatics (Oxford, England), 22(21), pp.26882690.

Stelkens, R. \& Seehausen, O., 2009. Genetic distance between species predicts novel trait expression in their hybrids. Evolution; international journal of organic evolution, 63(4), pp.884-897.

Stukenbrock, E.H. \& McDonald, B.A., 2008. The origins of plant pathogens in agro-ecosystems. Annual Review of Phytopathology, 46, pp.75-100.

Stukenbrock, E.H. et al., 2012. Fusion of two divergent fungal individuals led to the recent emergence of a unique widespread pathogen species. Proceedings of the National 
722

723

724

725

726

727

728

729

730

731

732

733

734

735

736

737

738

739

740

741

Taylor, A.L. \& Sasser, J.N., 1978. Biology, identification and control of root-knot nematodes (Meloidogyne species). International Meloidogyne Project, Raleigh, North.

Tigano, M.S. et al., 2005. Phylogeny of Meloidogyne spp. based on $18 \mathrm{~S}$ rDNA and the intergenic region of mitochondrial DNA sequences. Nematology, 7(6), pp.851-862.

Triantaphyllou, A.C., 1982. Cytogenetics and sexuality of root-knot and cyst nematodes. In R. D. Riggs, ed. Nematology in the southern region of the United States. Arkansas Agricultural Experiment Station, Fayetteville, AK: Southern Cooperative Series Bulletin, pp. 71-76.

Triantaphyllou, A.C., 1985. Cytogenetics, cytotaxonomy and phylogeny of root-knot nematodes. In J. N. Sasser \& C. C. Carter, eds. An Advanced Treatise on Meloidogyne. North Carolina State University, Raleigh.

Trudgill, D.L. \& Blok, V.C., 2001. Apomictic, polyphagous root-knot nematodes: Exceptionally successful and damaging biotrophic root pathogens. Annual Review of Phytopathology, 39, pp.53-77.

White, M.J.D., 1945. Animal Cytology \& Evolution 1st ed, Cambridge University Press.

Zerbino, D.R., 2010. Using the Velvet de novo assembler for short-read sequencing technologies. Current protocols in bioinformatics, Chapter 11, p.Unit 11.5.

Zerbino, D.R. \& Birney, E., 2008. Velvet: algorithms for de novo short read assembly using de Bruijn graphs. Genome Research, 18(5), pp.821-829.

Zhang, Z. et al., 2000. A greedy algorithm for aligning DNA sequences. Journal of computational biology : a journal of computational molecular cell biology, 7(1-2), pp.203-214. 


\section{Figure 1}

The relationships of tropical apomict Meloidogyne

This cartoon summarizes the relationships of the tropical apomict Meloidogyne root knot nematodes ("Group 1") to other Meloidogyne. Meloidogyne floridensis is a Group 1 species that can reproduce by meiotic parthenogenesis (blue colouration) while all other Group 1 species are obligate mitotic parthenogens (red colouration). Meloidogyne hapla is a meiotic parthenogenic species in Group 2. We have not used bifurcating trees to represent the relationships within the Group 1 and 2 species because of issues (highlighted in this paper) concerning possible hybrid origins of some taxa. 


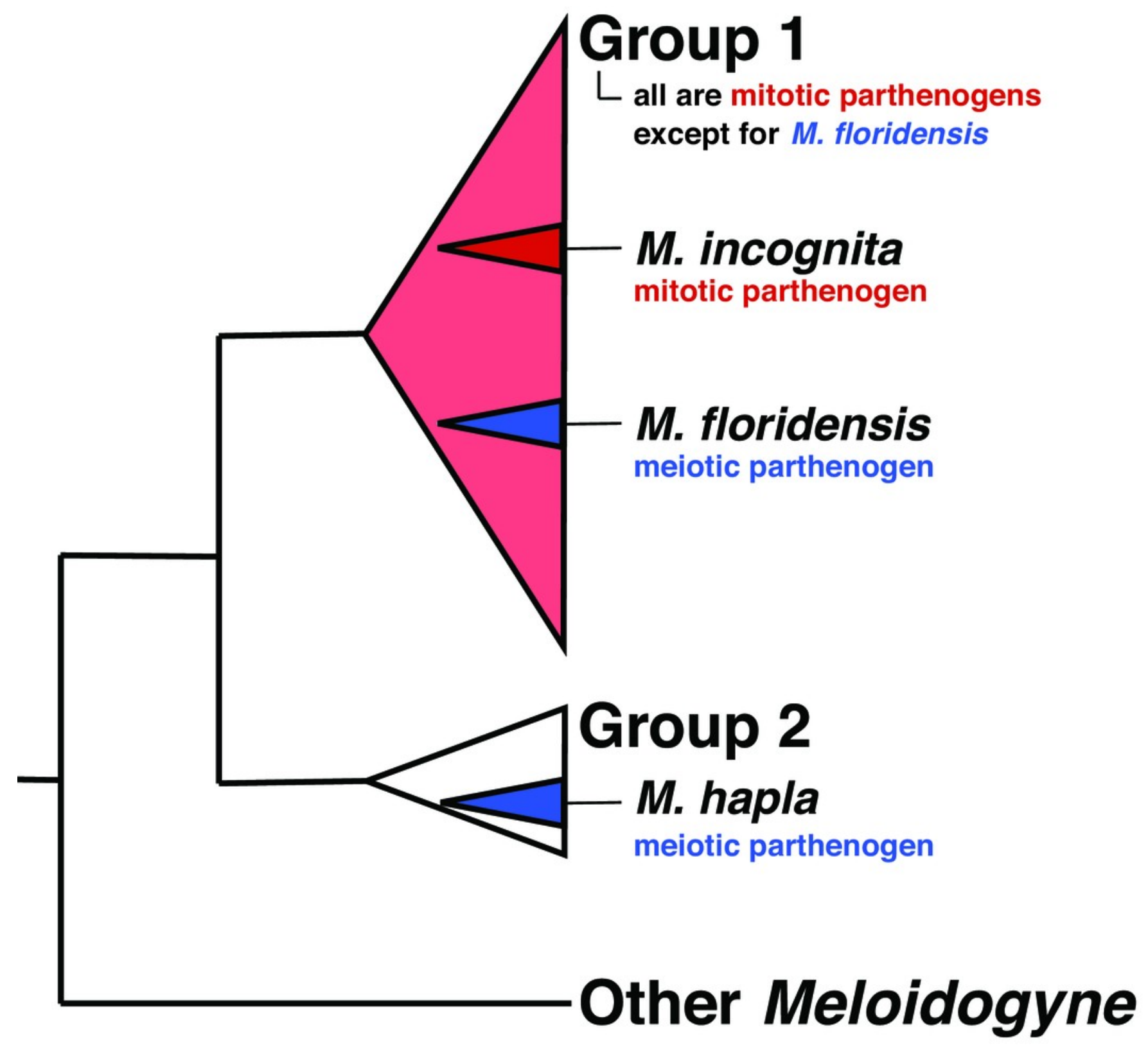




\section{Figure 2}

Scenarios of the possible relationships between Meloidogyne floridensis, Meloidogyne incognita and Meloidogyne hapla, and the origins of duplicated gene copies

M. hapla is a diploid species in a different sub-generic group to that of $M$. incognita and $M$. floridensis. Species " $\mathrm{X}$ ", " $Y$ " and "Z" are postulated ancestral parents that could have given rise to $M$. incognita and M. floridensis. A. Scenarios 1 and 2: Here M. floridensis is a diploid sister species to $M$. incognita and possesses the "X" genome. Scenario 1 postulates reacquisition of apomixis in M. floridensis from an apomict ancestor, while Scenario 2 postulates that the apomicts repeatedly lost meiosis independently. Under both these scenarios, the presence of significant duplications in $M$. incognita suggests that it has undergone whole genome endoduplication. The duplicated genomes (" $Z+Z$ ") in $M$. incognita are diverging under Muller's ratchet. B. Scenario 3: Ancestor "X" gave rise to the diploid species $M$. floridensis, and also interbred with " $Z$ " to yield $M$. incognita, which thus carries two divergent copies of each gene (" $\mathrm{X}+Z$ "). In this model only $M$. incognita, not $M$. floridensis, is predicted to carry two homeologues of many genes. C. Scenario 4: Both M. floridensis (" $\mathrm{X}+\mathrm{Y}$ ") and $M$. incognita (" $Y+Z$ ") are hybrid species, and share one parent (" $Y$ "). In this model both $M$. incognita and $M$. floridensis are predicted to carry two homeologues of many genes. D. Scenario 5: Both M. floridensis (" $\mathrm{X}+\mathrm{Y}$ ") and $M$. incognita (" $\mathrm{X}+\mathrm{Y}+\mathrm{Z}$ ") are hybrid species, but $M$. incognita is a triploid hybrid between the hybrid $M$. floridensis ancestor (" $X+Y$ ") and another species ("Z"). In this model M. incognita is predicted to carry three, and M. floridensis is predicted to carry two, homeologues of many genes. 


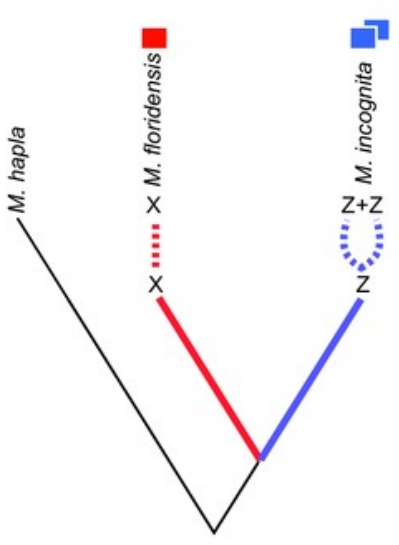

A M. incognita underwent whole genome duplication [scenarios 1 and 2]

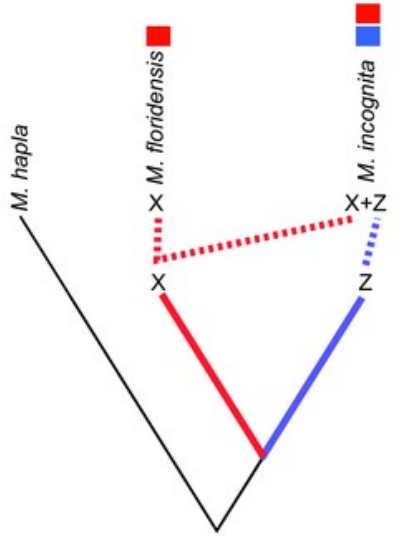

B $M$. incognita is an interspecific hybrid, with $M$. floridensis as one parent [scenario 3]

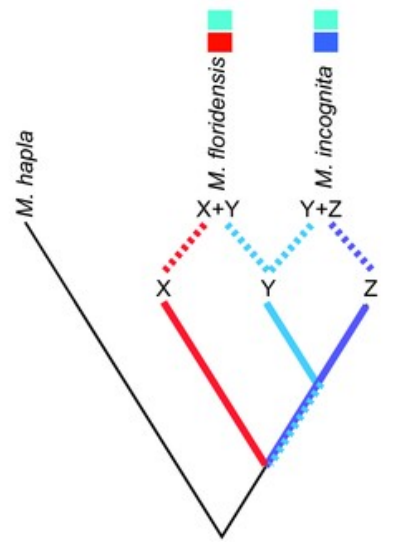

C M. floridensis and M. incognita are independent hybrids,

sharing one parent

[scenario 4]

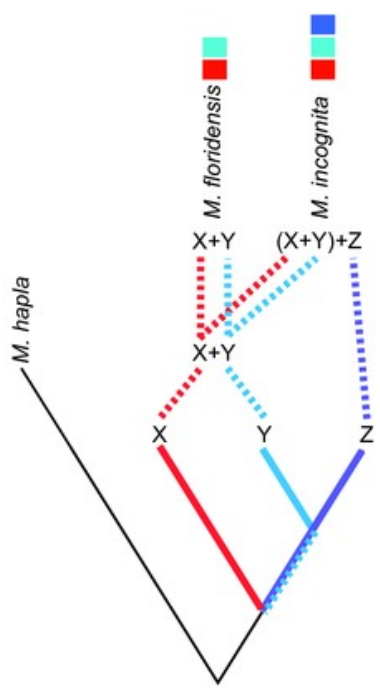

D M. floridensis is a hybrid, and $M$. incognita is a secondary hybrid between $M$. floridensis and a third parent [scenario 5] 


\section{Figure 3}

Inter- and intra-genomic identification of duplicated protein-coding regions

A Each coding sequence from each of the three target genomes (M. hapla, M. incognita and M. floridensis) was compared to the set of genes from the same species. The percent identity of the best matching (non-self) coding sequence was calculated, and is plotted as a frequency histogram. Both $M$. incognita and $M$. floridensis show evidence of the presence of many duplicates, while $M$. hapla does not. B The $M$. incognita gene predictions were compared to the M. floridensis genome and the M. hapla gene set. For each M. incognita gene, the similarity of the top matches in each genome was assessed. $M$. incognita has many genes that are highly similar to those of $M$. floridensis (similarity $>98 \%$ ). This contrasts with the matches to M. hapla, where the modal similarity is $~ 92 \%$, and there is no peak of high-similarity matches.
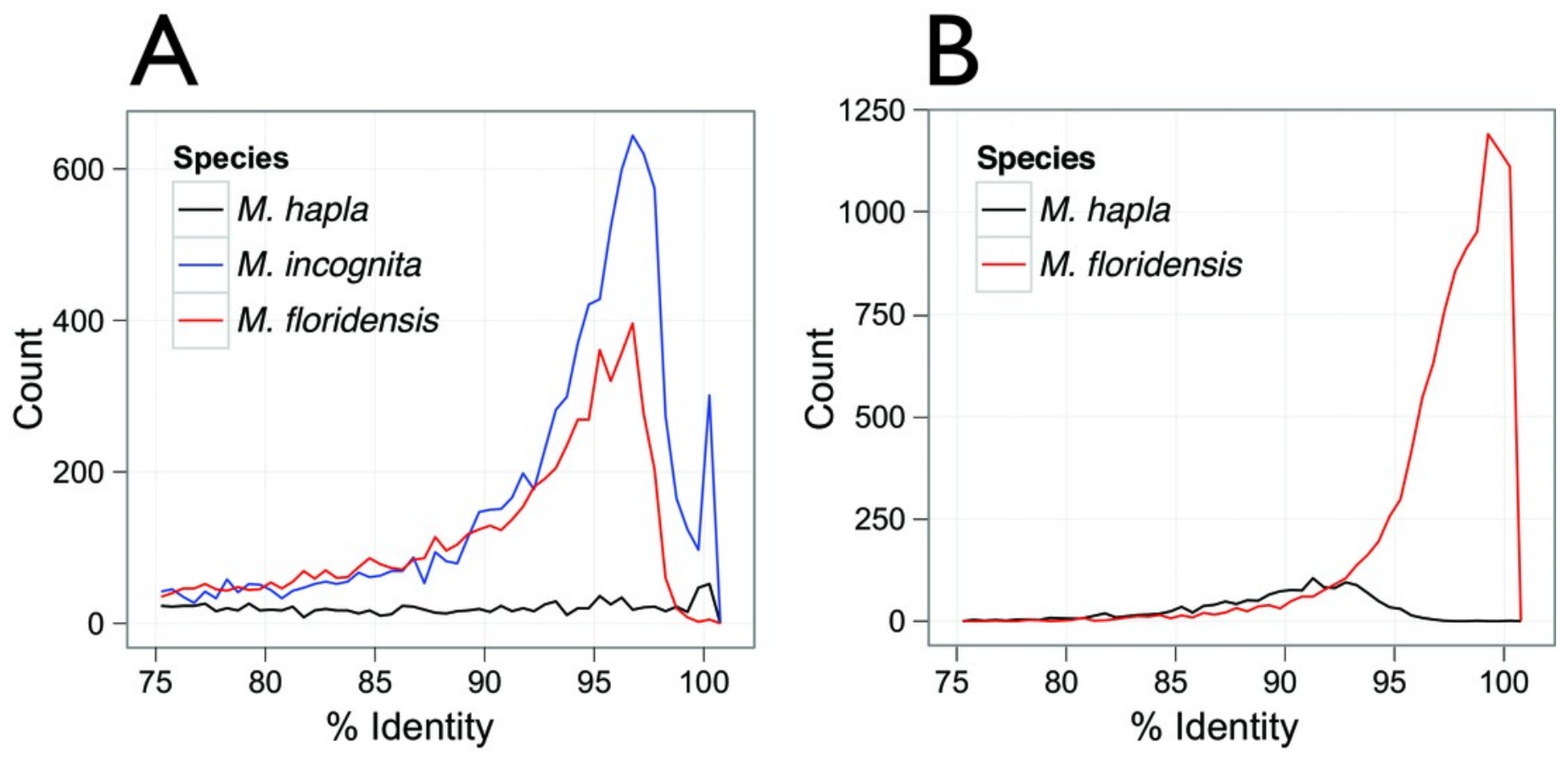


\section{Figure 4}

Phylogenomic analyses of clustered gene sets

For cluster sets represented in Table 2 that had representation of both $M$. floridensis and $M$. incognita, more than three members (i.e. where there was more than one possible topology), and fewer than five total members (i.e. where the number of possible topologies was still reasonably low and close to the number of clusters to be analyzed), we generated an estimate of the relationships between the sequences using RAxML. The resultant trees were bootstrapped, and rooted using the $M$. hapla representative. For each cluster set, the topologies were summarized by the different unique patterns possible. Within each figure cell, each cladogram in the figure is scaled by the number of clusters that returned that topology, with terminal nodes coloured by the origin of the sequences (black representing $M$. hapla, blue M. incognita, and red $M$. floridensis). The number of clusters congruent with each cladogram is given above the trees. The numbers of clusters contributing to each cell in the figure is represented by the grey box, which is scaled by the number of clusters summarized (e.g. the box in the central cell represents 902 trees, while the box in the bottom left cell represents 17 trees). 


\section{Table 1 (on next page)}

Summary statistics describing genome assemblies of Meloidogyne 


\begin{tabular}{|l|l|l|l|}
\hline Species & Meloidogyne hapla & $\begin{array}{l}\text { Meloidogyne } \\
\text { incognita }\end{array}$ & $\begin{array}{l}\text { Meloidogyne } \\
\text { floridensis }\end{array}$ \\
\hline Source & $\begin{array}{l}\text { NCSU / WormBase } \\
\text { WS227 }\end{array}$ & $\begin{array}{l}\text { INRA / WormBase } \\
\text { WS227 }\end{array}$ & $\begin{array}{l}\text { 959 Nematode } \\
\text { Genomes Project }\end{array}$ \\
\hline Data URL & $\begin{array}{l}\text { ftp://ftp.wormbase. } \\
\text { org/pub/wormbase/ } \\
\text { species/m_hapla/ }\end{array}$ & $\begin{array}{l}\text { ftp://ftp.wormbase.or } \\
\text { g/pub/wormbase/spe } \\
\text { cies/m_incognita/ }\end{array}$ & $\begin{array}{l}\text { http://downloads.ne } \\
\text { matodegenomes.or } \\
\text { g }\end{array}$ \\
\hline Citation & $\begin{array}{l}\text { (Opperman et al. } \\
2008)\end{array}$ & (Abad et al. 2008) & this work \\
\hline Maximum scaffold length & 360,446 & 154,116 & 40,762 \\
\hline Number of scaffolds & 3,452 & 9,538 & 81,111 \\
\hline Assembled size (bp) & $53,017,507$ & $82,095,019$ & $99,886,934$ \\
\hline Scaffold N50* (bp) & 37,608 & 12,786 & 3,516 \\
\hline GC\% & 27.4 & 31.4 & 29.7 \\
\hline CEGMA** completeness & $92.74 / 94.35$ & $75.00 / 77.82$ & $60.08 / 72.18$ \\
\hline Full / Partial & & $20,359(17,999)$ & 15,327 (15,121) \\
\hline $\begin{array}{l}\text { Predicted proteins (used } \\
\text { for clustering** })\end{array}$ & 13,072 (12,229) & & \\
\hline
\end{tabular}

* N50: weighted median contig length; the contig length at which $50 \%$ of the assembled genome is present in contigs of that or greater length.

** CEGMA: Core Eukaryotic Genes Mapping Approach (Parra et al. 2007).

*** Predicted proteins used for clustering and inferring phylogenies after filtering for length $>50$ amino acids (see Methods). 


\section{Table 2 (on next page)}

Numbers of Meloidogyne floridensis and Meloidoigyne incognita members in homeologue gene sets that have one Meloidogyne hapla member 


\begin{tabular}{|l|l|l|l|l|l|}
\hline & $\begin{array}{l}0 \mathrm{M} . \\
\text { incognita } \\
\text { members }\end{array}$ & $\begin{array}{l}1 \mathrm{M} . \\
\text { incognita } \\
\text { member }\end{array}$ & $\begin{array}{l}2 \mathrm{M} . \\
\text { incognita } \\
\text { members }\end{array}$ & $\begin{array}{l}3 \mathrm{M} . \\
\text { incognita } \\
\text { members }\end{array}$ & $\begin{array}{l}\text { incognita } \\
\text { members }\end{array}$ \\
\hline $\begin{array}{l}0 \text { M. floridensis } \\
\text { members }\end{array}$ & 0 & 907 & 327 & 44 & 17 \\
\hline $\begin{array}{l}1 \mathrm{M} . \text { floridensis } \\
\text { member }\end{array}$ & 2196 & 2189 & 920 & 102 & 40 \\
\hline $\begin{array}{l}2 \text { M. floridensis } \\
\text { members }\end{array}$ & 226 & 257 & 156 & 36 & 21 \\
\hline $\begin{array}{l}3 \text { M. floridensis } \\
\text { members }\end{array}$ & 17 & 17 & 20 & 7 & 14 \\
\hline $\begin{array}{l}>3 \text { M. } \\
\text { floridensis } \\
\text { members }\end{array}$ & 8 & 11 & 6 & 4 & 21 \\
\hline
\end{tabular}

